\title{
Correlation of local structure and diffusion pathways in the modulated anisotropic oxide ion conductor $\mathrm{CeNbO}_{4.25}$
}

Stevin S. Pramana, ${ }^{\mathrm{a}}$ Tom Baikie, ${ }^{\mathrm{b} *}$ Tao An,${ }^{\mathrm{c}}$ Matthew G. Tucker, ${ }^{\mathrm{d}, \mathrm{e}} \mathrm{Ji}$ Wu, ${ }^{\mathrm{a}}$ Martin K. Schreyer, ${ }^{\mathrm{f}}$ Fengxia Wei, ${ }^{\mathrm{g}}$ Ryan D. Bayliss, ${ }^{\mathrm{a}}{ }^{\dagger}$ Christian L. Kloc, ${ }^{\mathrm{c}}$ Timothy J. White,${ }^{\mathrm{c}}$ Andrew P. Horsfield, ${ }^{\mathrm{a}}$ and Stephen J. Skinner. ${ }^{a^{*}}$

a Department of Materials, Imperial College London, Exhibition Road, London, SW7 2AZ, United Kingdom

b Energy Research Institute @ NTU (ERI@N), Research Technoplaza, Nanyang Technological University, Nanyang Drive, Singapore

c Division of Materials Science and Engineering, Nanyang Technological University, Singapore

d ISIS Facility, Rutherford Appleton Laboratory, Didcot OX11 0QX, United Kingdom

e Diamond Light Source, Chilton, Oxfordshire, OX11 0DE, United Kingdom

${ }^{\mathrm{f}}$ Institute of Chemical and Engineering Sciences, 1 Pesek Road, Jurong Island, Singapore

${ }^{\mathrm{g}}$ Institute of Materials Research and Engineering, 3 Research Link, Singapore

*e-mail: tbaikie@ntu.edu.sg, s.skinner@imperial.ac.uk

$\dagger$ Present address: Department of Chemistry, University of Illinois at Chicago, 845 W. Taylor Street (MC 111), Chicago, Illinois, 60607, USA 


\begin{abstract}
$\mathrm{CeNbO}_{4.25}$ is reported to exhibit fast oxygen ion diffusion at moderate temperatures, making this the prototype of a new class of ion conductor with applications in a range of energy generation and storage devices. To date, the mechanism by which this ion transport is achieved has remained obscure, in part due to the long range commensurately modulated structural motif. Here we show that $\mathrm{CeNbO}_{4.25}$ forms with a unit cell $\sim 12$ times larger than the stoichiometric tetragonal parent phase of $\mathrm{CeNbO}_{4}$ as a result of the helical ordering of $\mathrm{Ce}^{3+}$ and $\mathrm{Ce}^{4+}$ ions along $z$. Interstitial oxygen ion incorporation leads to a cooperative displacement of the surrounding oxygen species creating inter-layer " $\mathrm{NbO}_{6}$ " connectivity by extending the oxygen coordination number to 7 and 8 . Molecular dynamic simulations suggest that fast ion migration occurs predominantly within the $x z$ plane. It is concluded that the oxide ion diffuses anisotropically, with the major migration mechanism being intra-layer; however when obstructed, oxygen can readily move to an adjacent layer along y via alternate lower energy barrier pathways.
\end{abstract}

\title{
Keywords
}

Mixed ionic-electronic conductor, Crystallography, Pair distribution function, Single crystal, Molecular dynamics, Ion transport. 


\section{INTRODUCTION}

Materials with oxygen hyper-stoichiometry have recently gained importance in the field of solid oxide electrochemical cells due to their low activation energy for the motion of interstitial oxide ions, as for example, in doped and undoped lanthanide silicate and germanate apatites, ${ }^{1}$ melilites such as $\mathrm{La}_{1+x} \mathrm{Sr}_{x} \mathrm{Ga}_{3} \mathrm{O}_{7+x / 2}{ }^{2,3}$ as well as oxides such as $\mathrm{Ce}(\mathrm{V}, \mathrm{Ta}) \mathrm{O}_{4+\delta}{ }^{4-7}$ Interstitial ion transport offers an alternative conduction mechanism to the vacancy-transport found in highsymmetry three-dimensional mixed ionic electronic conductors such as $\mathrm{La}_{1-x} \mathrm{Sr}_{x}(\mathrm{Co}, \mathrm{Mn}, \mathrm{Fe}) \mathrm{O}_{3}$ $\delta$ perovskite ${ }^{8}$ and the ionic $\mathrm{Zr}_{1-x} \mathrm{Y}_{x} \mathrm{O}_{2-\delta}$ fluorite materials, ${ }^{9}$ and could overcome the perceived requirement for high symmetry in new ion conducting materials. One of the early attempts to depart from vacancy-based transport was the development of the interstitialcy mechanism, as reported for the mixed conducting $(\mathrm{La}, \mathrm{Pr})_{2}(\mathrm{Ni}, \mathrm{Co}) \mathrm{O}_{4+\delta}$ Ruddlesden-Popper phases. ${ }^{10,11}$ One of the more promising prototypical materials currently under investigation is low symmetry $\left[\mathrm{Ce}^{3+}{ }_{1-2 \delta} \mathrm{Ce}^{4+}{ }_{2 \delta}\right] \mathrm{NbO}_{4+\delta}$, which is a mixed ionic and $p$-type electronic conductor at oxygen chemical potentials close to atmospheric (total conductivity, $\sigma_{\text {total }}=0.030 \mathrm{~S} \mathrm{~cm}^{-1}$ at $850^{\circ} \mathrm{C}$; ion transference number $=0.4$; diffusion coefficient, $\left.D=8.3 \times 10^{-8} \mathrm{~cm}^{2} \mathrm{~s}^{-1}\right) .{ }^{12-16}$ The oxygen diffusivity is higher than the common electronic conductor $\mathrm{La}_{1-x} \mathrm{Sr}_{x} \mathrm{MnO}_{3-\delta}$, and comparable with the mixed-conducting $\mathrm{La}_{1-x} \mathrm{Sr}_{x} \mathrm{CoO}_{3-\delta}$, analogue despite possessing a slightly higher activation energy. ${ }^{8,15}$ At ambient temperature four different crystal structures with varying oxygen contents have been identified in this family of materials corresponding to the formulae $\mathrm{CeNbO}_{4}, \mathrm{CeNbO}_{4.08}, \mathrm{CeNbO}_{4.25}$ and $\mathrm{CeNbO}_{4.33}{ }^{17,18}$ In order to better understand the oxygen transport mechanism and optimize performance, defect crystallography needs to be fully explored. The parent structure $\mathrm{CeNbO}_{4}$, which does not contain any interstitial ions, was shown by powder neutron diffraction data to be a monoclinic fergusonite-type structure at room temperature, ${ }^{19,20}$ that transforms to a stoichiometric tetragonal polymorph (scheelite-type) at $\geq$ 
$800^{\circ} \mathrm{C} .{ }^{15} \mathrm{We}$ have recently re-determined the structure of $\mathrm{CeNbO}_{4}$ from single crystal X-ray diffraction, ${ }^{20}$ whilst to date none of the hyper-stoichiometric phases showing higher ionic conductivity than the stoichiometric $\delta=0$ phases, have solved crystal structures. ${ }^{21,22}$ However electron microscopy studies found alternating commensurate and incommensurate modulated structures, ${ }^{16}$ which arise from the incorporation of excess oxygen on interstitial sites. In these cases charge balance is maintained by oxidation of $\mathrm{Ce}^{3+}$ to $\mathrm{Ce}^{4+} \cdot{ }^{12,14,23}$ All previous structural refinements have used powdered samples; however, the Rietveld refinement of powder X-ray diffraction data for modulated structures with large cells or incommensuration is challenging and can lead to unconvincing refinements and/or ambiguous results. This is due to the large number of calculated reflections, high reflection overlap and often, weak supercell or incommensurate reflections. In order to minimize these issues, large single crystals of the parent structure were prepared, and subsequent post heat treatment of these crystals in appropriate oxidising environments led to the formation of the $\delta>0$ phases (Figure S1). The long-range average structure was modelled by analysing the neutron and synchrotron X-ray powder diffraction data and short-range coordination environment was determined using pair distribution function (PDF) analysis of neutron diffraction data obtained from powdered samples, prepared as outlined in our previous work..$^{20}$ 


\section{METHODS}

\section{Experimental}

Commercial powders of $\mathrm{CeO}_{2}$ (Merck, 99.9\%) and $\mathrm{Nb}_{2} \mathrm{O}_{5}$ (Sigma Aldrich, 99.99\%) were used as starting materials, mixed in stoichiometric proportions and calcined in a box furnace at $1273 \mathrm{~K}$ in static air for 12 hours. After grinding, the powder was pressed under an isostatic pressure of $100 \mathrm{MPa}$ to prepare two cylindrical rods with approximate diameter of $6 \mathrm{~mm}$ and length of $40 \mathrm{~mm}$, which were subsequently sintered at $1673 \mathrm{~K}$ for $16 \mathrm{~h}$ in air. The growth apparatus utilises an optical floating zone furnace with four ellipsoidal mirrors in which 700W halogen lamps were set as the infrared source with the growth rate of $10 \mathrm{~mm} / \mathrm{h}$, counter rotation rate of $40 \mathrm{rpm}$, and in flowing argon gas atmosphere. To prepare $\mathrm{CeNbO}_{4.25}$ from the parent crystal $\mathrm{CeNbO}_{4}$, a crystal with approximate dimensions $5 \times 5 \times 5 \mathrm{~mm}$ was heated in a box furnace in static air at $873 \mathrm{~K}$ for $96 \mathrm{~h}$ and then quenched to room temperature. The phase purity of the parent and oxidised phases were confirmed using laboratory powder X-ray diffraction. The latter crystal was cleaved for analysis by single crystal X-ray diffraction, collected at room temperature with a Bruker Smart Apex II three-circle diffractometer using graphite monochromated $\mathrm{MoK}_{\alpha}$ radiation over $5 \leq 2 \theta \leq 55^{\circ}$. An empirical absorption correction was applied and the data were refined using Jana $2006^{24}$ utilising the Superflip ${ }^{25}$ structure solution algorithm. The atomic positions and anisotropic displacement parameters (ADPs) for $\mathrm{Ce}, \mathrm{Nb}$ and $\mathrm{O}$ were refined. Powders with the nominal composition of $\mathrm{CeNbO}_{4.25}$ were analysed using high brilliance synchrotron radiation at I11 (Diamond Light Source, Rutherford Appleton Laboratory, Didcot, UK, $\lambda=0.826471 \AA$ ) . The crystallographic model used was that derived from the single crystal XRD data. Chebyshev polynomial coefficients, scale, lattice parameters and spherical harmonics anisotropic peak broadening were sequentially refined using TOPAS $V 4.1^{26}$. Isotropic atomic displacement parameters were refined in groups for $\mathrm{Ce}, \mathrm{Nb}$ and $\mathrm{O}$ with 
the atomic fractional coordinate fixed. Neutron powder diffraction was conducted at the D2B beamline (Institut Laue-Langevin, Grenoble, France) using a wavelength of $1.594 \AA$. The same model as used for the synchrotron powder XRD was used to fit the neutron powder diffraction data and the same strategy used to refine the synchrotron powder XRD was applied, excluding the spherical harmonics anisotropic peak broadening factor. Total scattering neutron powder diffraction data were collected from the Polaris beamline at ISIS, Rutherford Appleton Laboratory, UK, up to a value of $29.5 \AA^{-1}$ in the scattering vector $Q=4 \pi \sin \theta / \lambda$. Background data from the instrument and sample holder were also collected and subtracted. The neutron diffraction data for Bragg scattering were analysed using $\mathrm{GSAS}^{27}$ and the total scattering data correction was performed using Gudrun ${ }^{28}$ before the Reverse Monte Carlo ${ }^{29,30}$ analysis adopted in RMCProfile. ${ }^{31}$ For the $\mathrm{CeNbO}_{4}$ data the model was based on a 10x10x10 supercell $(24000$ atoms) based on the published structure ${ }^{19}$ whilst for the $\mathrm{CeNbO}_{4.25}$ a $4 \mathrm{x} 4 \mathrm{x} 4$ supercell, using the structure derived in this work, was selected ensuring an ensemble of 19200 atoms were included. Powders for electron diffraction were prepared by grinding under ethanol and drops of the resulting suspension deposited on lacey-carbon coated copper-grids. Images were collected using a $200 \mathrm{kV}$ JEOL-2100F microscope with a low-background Gatan double tilt holder. Field-limiting apertures for selected-area electron diffraction (SAED) were 5, 20 and $60 \mu \mathrm{m}$ in diameter. Crystals were tilted to [010] and [201] zone axes. High resolution images were collected using an objective aperture $(100 \mu \mathrm{m})$, corresponding to a nominal point-to-point resolution of $\sim 1.7 \AA$ A Electron diffraction patterns were calibrated against external standards to derive reliable values for both the electron wavelength and camera length. Lattice parameters were determined repeatedly to check for hysteresis of the electromagnetic lenses leading to errors $< \pm 1 \%$. 


\section{Computational Methods}

To study the diffusion dynamics of the oxygen ions in $\mathrm{CeNbO}_{4}$ and $\mathrm{CeNbO}_{4.25}$, molecular dynamics (MD) simulations based on a Born-like description ${ }^{32}$ of the ionic lattices were performed. The interactions between ions were represented by long-range Coulombic force (summed using Ewald's method ${ }^{33}$ ) and the short-range Buckingham pair potential. ${ }^{34}$ The cutoff distance for the summation of the Buckingham pair potential was $10.5 \AA$, as it is significant only at short range. The lattice energy is then given by

$$
E_{\text {lattice }}=\sum_{j>i} \sum\left[\frac{q_{i} q_{j}}{4 \pi \varepsilon_{0} r_{i j}}+A_{i j} \exp \left(-\frac{r_{i j}}{\rho_{i j}}\right)-\left(\frac{C_{i j}}{r_{i j}^{6}}\right)\right]
$$

where $r_{i j}$ is the distance between ion $i$ and $j, q_{i(j)}$ is the charge of ion $i(j), A_{i j}, \rho_{i j}$ and $C_{i j}$ are the Buckingham pair potential parameters (listed in Table S4) and $\varepsilon_{0}$ is the permittivity of free space. The starting crystal geometries used in the simulations were based on our previous measured values for $\mathrm{CeNbO}_{4}{ }^{20}$ and this work for $\mathrm{CeNbO}_{4.25}$. The supercells used to construct the periodic lattices were of $10 \times 10 \times 8$ and $4 \times 4 \times 4$ unit cells for $\mathrm{CeNbO}_{4}$ and $\mathrm{CeNbO}_{4.25}$ respectively with periodic boundary conditions applied. Both supercells contained 19200 ions. All molecular dynamics simulations were performed using the DL_POLY4 package ${ }^{35,36}$ with the velocity Verlet integration algorithm. ${ }^{37}$ The oxygen diffusion behaviour was studied in the temperature range of 573-2173K. The lattices were first relaxed using GULP (General Utility Lattice Program). ${ }^{38}$ The relaxed supercell was equilibrated at constant volume (NVT) for $10 \mathrm{ps}$ and then re-equilibrated at constant pressure (NPT), again for 10 ps. The lattice energies, volumes and temperatures were checked to ensure equilibrium was reached. Diffusion dynamics data were collected for $\sim 100$ ps using the Nosé-Hoover thermostat. The visual molecular dynamics (VMD) package was used to analyze the data. ${ }^{39}$ The production run for this work was undertaken in NVT ensemble. The first 60ps of data have been excluded from 
analysis to ensure equilibrium was reached. Table S5 lists the calculated lattice parameters for both systems showing good agreement with the experimentally measured lattice parameters. 


\section{RESULTS AND DISCUSSION}

The selected area electron diffraction (SAED) pattern of single crystal $\mathrm{CeNbO}_{4.25}$, obtained from oxidising $\mathrm{CeNbO}_{4}$, shows a commensurately modulated phase compared to the parent phase (Figure 1). Along $[010]_{\mathrm{p}}$ zone axis (Figure 1a), the resultant superstructure reciprocal space unit cell was determined to be:

$$
\begin{aligned}
& \mathbf{a}_{\mathrm{r}}^{*}=\frac{1}{12}[204]_{\mathrm{p}}^{*} \\
& \mathbf{c}_{\mathrm{r}}^{*}=\frac{1}{6}[\overline{2} 02]_{\mathrm{p}}^{*}
\end{aligned}
$$

where $\mathrm{p}$ and $\mathrm{r}$ represent the parent and resultant superstructure cell, respectively.
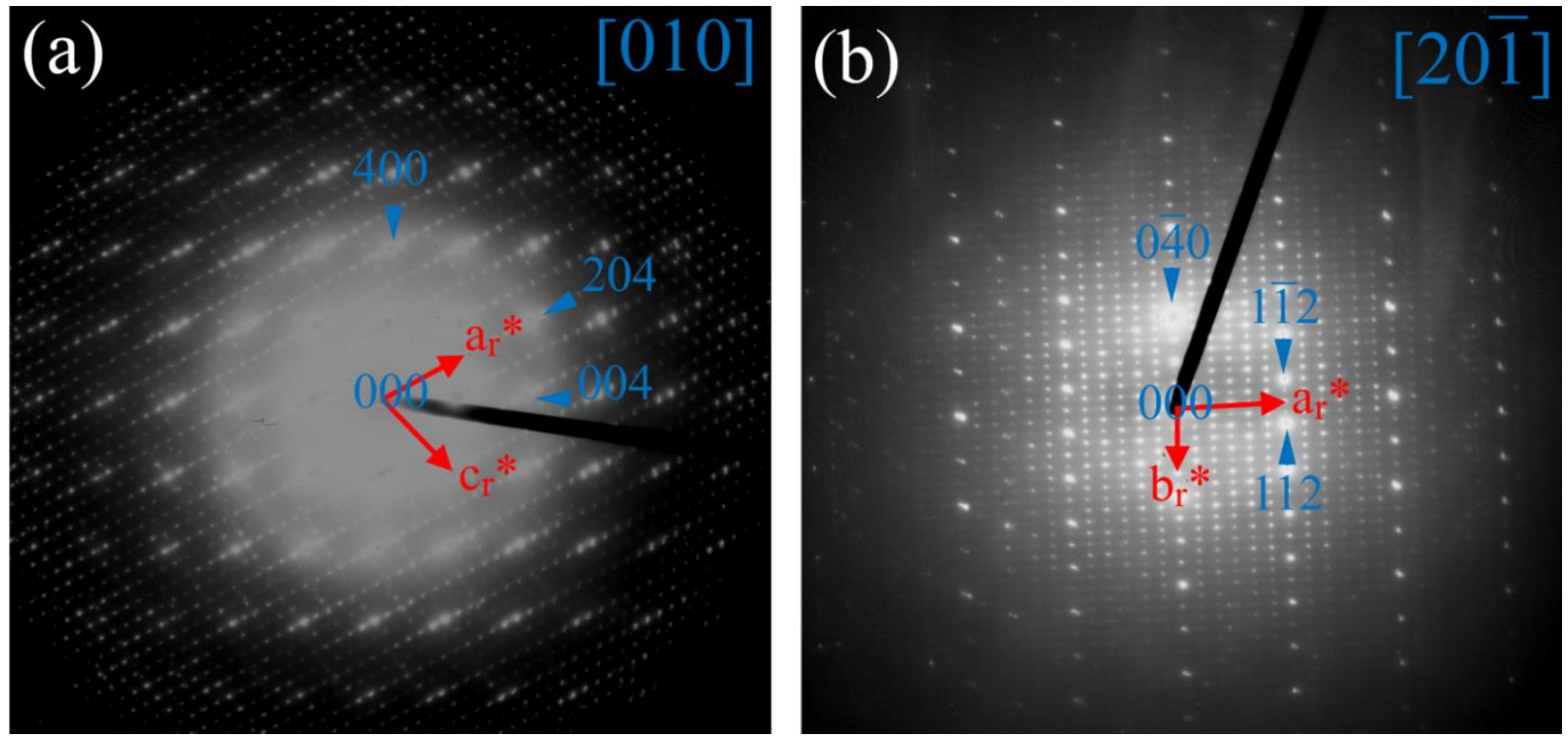

Figure 1: Selected area electron diffraction (SAED) of a CeNbO4.25 crystal oriented along

(a) $[010]_{p}$ and (b) $[20 \overline{1}]_{p}$ showing a superstructure in $P 2_{1} / c$ with the reciprocal space axes marked by red arrows. All indices are with respect to the parent substructure of $\mathrm{CeNbO}_{4}$ crystallized in I2/a. 
In addition, to determining the unique $\mathbf{b}_{\mathrm{r}}{ }^{*}$, the crystal was oriented perpendicular to the first zone axis along $[20 \overline{1}]_{\mathrm{p}}$ zone axis, and it was concluded that $\mathbf{b}_{\mathrm{r}}^{*}=\frac{1}{4}[020]_{\mathrm{p}}^{*}$ (Figure $1 \mathrm{~b}$ ) resulted in the space group $P 2{ }_{1} / c$ as suggested previously. ${ }^{16}$ Closer inspection of Figure $1 \mathrm{~b}$ shows weak diffuse streaking along $\mathbf{a}_{\mathrm{r}}{ }^{*}$ consistent with stacking disorder. Hence, the resultant supercell real space unit cell derived from the observed reciprocal space is given by a matrix:

$$
\left(\begin{array}{l}
\mathbf{a}_{\mathrm{r}} \\
\mathbf{b}_{\mathrm{r}} \\
\mathbf{c}_{\mathrm{r}}
\end{array}\right)=\left(\begin{array}{ccc}
2 & 0 & 2 \\
0 & 2 & 0 \\
-2 & 0 & 1
\end{array}\right)\left(\begin{array}{l}
\mathbf{a}_{\mathrm{p}} \\
\mathbf{b}_{\mathrm{p}} \\
\mathbf{c}_{\mathrm{p}}
\end{array}\right)
$$

The single crystal X-ray diffraction reflections yielded a monoclinic cell (Space group $P 1{ }_{1} / c 1$; $a=14.3732(8) \AA, b=22.7921(12) \AA, c=11.8319(6) \AA$ and $\beta=105.071(2)^{\circ}$, Table S1) in good agreement with the cell previously determined by Thompson et. al. ${ }^{16}$ using TEM $\left(P 12_{1} / c 1\right.$ $a=14.371(2) \AA, b=22.748(6) \AA, c=11.820(2) \AA$ and $\beta=74.91(2)$ where $\beta$ is defined as the supplementary angle. Using 48 formula units per unit-cell $(Z=48) 12$ unique Ce and 12 unique $\mathrm{Nb}$ positions were readily found while 51 unique oxygen sites were determined from the difference Fourier maps calculated using the Jana2006 software package ${ }^{24}$ with the reliability indicators converging to acceptable values: $R=0.0431, R_{w}=0.0945, G O F=1.79$ (Table S2). In addition, bond distances and angles fall within the expected ranges for ionic interactions, confirming a high quality model.

Bond valence sums (BVS) ${ }^{40,41}$ which measure the 'bond strength' in ionic compounds using all interactions up to a distance of $3 \AA$ are shown in Table S3. Different valence states of the Ce ions were distinguished from the BVS analysis. As expected from charge balance considerations in $\mathrm{CeNbO}_{4.25}$, of the 12 unique Ce positions identified, six sites were found to contain $\mathrm{Ce}^{3+}$ and the remaining six were found to be $\mathrm{Ce}^{4+}$ sites. In the parent material, $\mathrm{CeNbO}_{4}$, 
all of the $\mathrm{Ce}^{3+}$ ions are coordinated to 8 oxygen ions as first nearest neighbour and all of the $\mathrm{Nb}^{5+}$ ions are coordinated to 4 and 2 oxygen ions as first and second nearest neighbours, respectively; in $\mathrm{CeNbO}_{4.25}$ the excess 0.25 oxygen per formula unit, equivalent to 12 extra oxygen ions in 3 unique Wyckoff sites, is expected to change the overall coordination environments of both ions significantly. From analysis of the BVS, on average $\mathrm{Ce}^{4+}$ ions were coordinated to fewer oxygen ions (8.67) when compared with $\mathrm{Ce}^{3+}$ (9.5) in $\mathrm{CeNbO}_{4.25}$, and significantly more than the 8 coordinate environment in stoichiometric $\mathrm{CeNbO}_{4}$, within a $3 \AA$ window. The higher coordination number could be achieved by adjusting the various $\mathrm{Ce}-\mathrm{O}$ bond lengths so that the correct bond valence sums are satisfied. The bond valence sums for all $\mathrm{Nb}$ ions are calculated to be close to 5+ with slight underbonding found for one $\mathrm{Nb}$ equivalent position. The $\mathrm{Nb}$ ions retain their 6 coordinate environment with the exception of sites labelled as $\mathrm{Nb}(1)$ and $\mathrm{Nb}(8)$ (refer to $\mathrm{SI}$ ), which enlarge to 8 and 7 coordinate respectively, resulting in an average of $\mathrm{NbO}_{6.25}$ for the first two nearest neighbour shells. This weakens oxygen binding energy to the niobium allowing mobile oxygen to migrate from one crystallographic site to another.

Figure $\mathrm{S} 2$ compares the parent $\mathrm{CeNbO}_{4}$ and oxidized $\mathrm{CeNbO}_{4.25}$ projected along the principal axes of [100], [010], [001] and [101]. Displacement of oxygen from the original positions in the parent structure on the $x z$ plane is observed. In order to simplify the structural representation, in $\mathrm{CeNbO}_{4}, \mathrm{NbO}_{6}$ polyhedra are continuously connected by edge-sharing in the $x z$ plane with stacking along the $y$ axis, creating a layered slab, while the incorporation of interstitial oxygen ions in $\mathrm{CeNbO}_{4.25}$ are clearly positioned by the cooperative displacement mechanism of the surrounding oxygen ions to satisfy the bond valence sum (Figures $2 a$ and $b$ ). Despite increasing the coordination number of 6 for $\mathrm{Nb}$ ions to 7 , giving $\mathrm{Nb}(8) \mathrm{O}_{7}$ and 8 giving $\mathrm{Nb}(1) \mathrm{O}_{8}$, the interstitial species are not located at the vacant $4 b$ Wyckoff site in the 
(a)

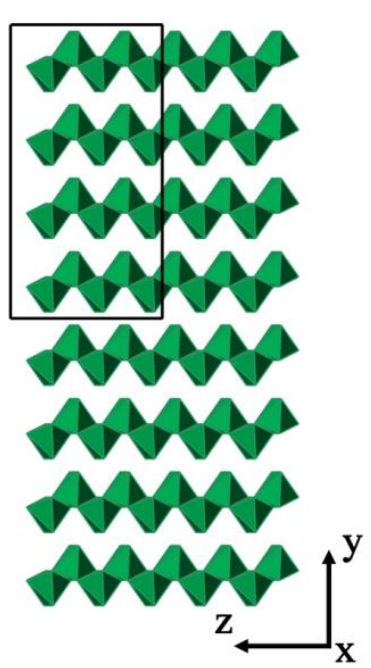

(d)

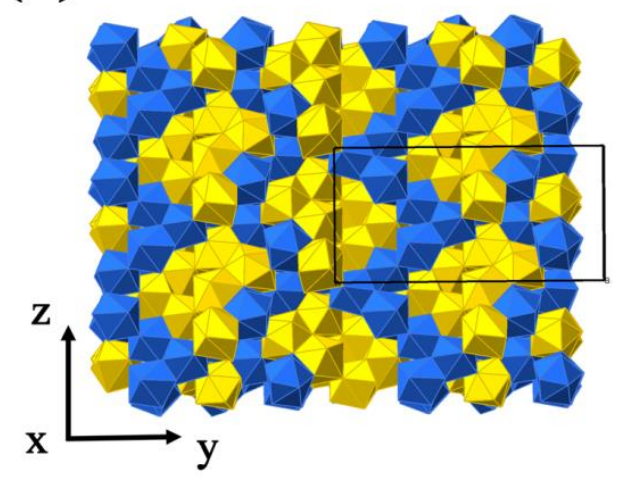

(b)

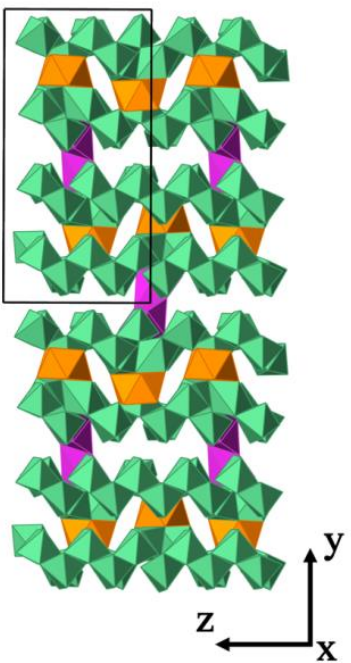

(c)

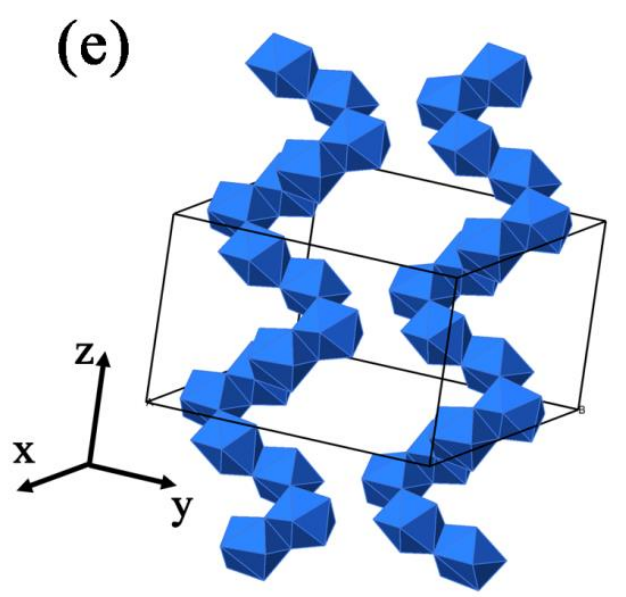

Figure 2: The structural arrangement of $\mathrm{NbO}_{6}$ (green), $\mathrm{NbO}_{7}$ (purple) and $\mathrm{NbO}_{8}$ (orange) projected along [100] in (a) $\mathrm{CeNbO}_{4}$ and (b) $\mathrm{CeNbO}_{4.25}$ while (c) illustrates connectivity between $\mathrm{NbO}_{\mathrm{x}}$ slabs in the oxidized $\mathrm{CeNbO}_{4.25}$ along $y$ built by the insertion of interstitial oxygen ions creating edge-shared $\mathrm{NbO}_{6}-\mathrm{NbO}_{7}-\mathrm{NbO}_{7}-\mathrm{NbO}_{6}$, corner-connected $\mathrm{NbO}_{6}$ - NbOs (orange) and edge-shared $\mathrm{NbO}_{8}$ - $\mathrm{NbO}_{6}$ polyhedra facilitating the improved conductivity, (d) The structural arrangement of $\mathrm{Ce}^{4+} \mathrm{O}_{\mathrm{x}}$ (blue) and $\mathrm{Ce}^{3+} \mathrm{O}_{\mathrm{x}}$ (yellow) polyhedra in $\mathrm{CeNbO}_{4.25}$ projected along the principal [100] and (e) $[-1.5,-0.7,-0.7]$. $\mathrm{Ce}^{4+}$ polyhedra are edge-shared to form the continuous helical network along $z$.

distorted superstructure of the fluorite-type $\mathrm{e}^{42,43}$ as suggested previously by Thompson et al. ${ }^{16}$ (Figure S3). In between the $\mathrm{NbO}_{6}$ slabs along the $y$ axis, the connectivity is built from sinusoidal bridging oxygens (Figure 2c), that is believed to facilitate enhanced ionic conductivity along the $x z$ plane whenever the mobile oxygen ions encounter a hindrance along the slab and alternatively conduct to the next layer perpendicular ( $y$ direction) to the slabs, 
similar to the inter-tunnel diffusion found in the apatite electrolytes in addition to the major intra-tunnel migration. ${ }^{44}$ Figures $2 \mathrm{~d}$ and e show solely the polyhedra of $\mathrm{Ce}^{4+} \mathrm{O}_{\mathrm{x}}$ (blue) and $\mathrm{Ce}^{3+} \mathrm{O}_{\mathrm{x}}$ (yellow) where the higher oxidation state $\mathrm{Ce}^{4+}$ species are found to form a continuous helical network running parallel to the crystallographic $z$ axis, connected by edge-sharing. In order to verify the average crystal structure of the powders, a Rietveld plot of synchrotron powder XRD and neutron diffraction patterns are presented in Figure S4 showing a good fit using the model derived from the single crystal XRD data. $R_{w p}, R_{B r a g g}$ and $\chi^{2}$ for the synchrotron XRD and neutron diffraction Rietveld fits were $0.097,0.035$ and 1.28; 0.034, 0.014 and 0.75, respectively.

In order to highlight differences in the local structure of the $\mathrm{Ce}$ and $\mathrm{Nb}$ environments pair distribution function analysis of $\mathrm{CeNbO}_{4}$ and $\mathrm{CeNbO}_{4.25}$ powders was performed with the reliability factors for the Bragg profile fits being 0.046 and 0.044 , respectively. The total scattering structure factor $\mathrm{S}(\mathrm{Q})$ and partial pair correlation functions $\mathrm{g}(\mathrm{r})$ of $\mathrm{Ce}-\mathrm{O}$ and $\mathrm{Nb}-\mathrm{O}$ with $r \leq 10 \AA$ are presented in Figure 3. The $\mathrm{g}(\mathrm{r})_{\mathrm{Nb}-\mathrm{O}}$ of $\mathrm{CeNbO}_{4}$ shows a separation between the first three coordination environments while the shoulder at $2 \leq \mathrm{r} \leq 3 \AA$ in the oxidized sample is consistent with the extended coordination of $\mathrm{Nb}-\mathrm{O}$ from 6 to 7 and 8 due to the insertion of three interstitial oxygen ions per formula unit. The Ce-O pair correlation function in the latter sample is also broader suggesting that the existence of mixed $\mathrm{Ce}^{3+}$ and $\mathrm{Ce}^{4+}$ ions must be facilitated by flexing the bond strength around the oxygen ion neighbours. The PDF histograms of the calculated $\mathrm{Ce}-\mathrm{O}$ and $\mathrm{Nb}-\mathrm{O}$ bond lengths are consistent with the data extracted from the single crystal X-ray diffraction (Figure 4). The close agreement between the structure obtained from powder and single crystal diffraction (long range), supported by 
the short-range coordination information yielded from the powder total scattering, validates the usefulness of the 'average' technique, should the single crystal be difficult to produce.

(a)

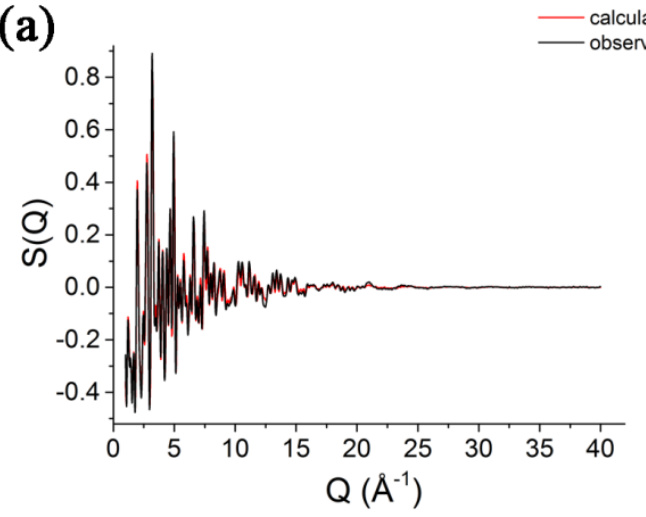

(c)

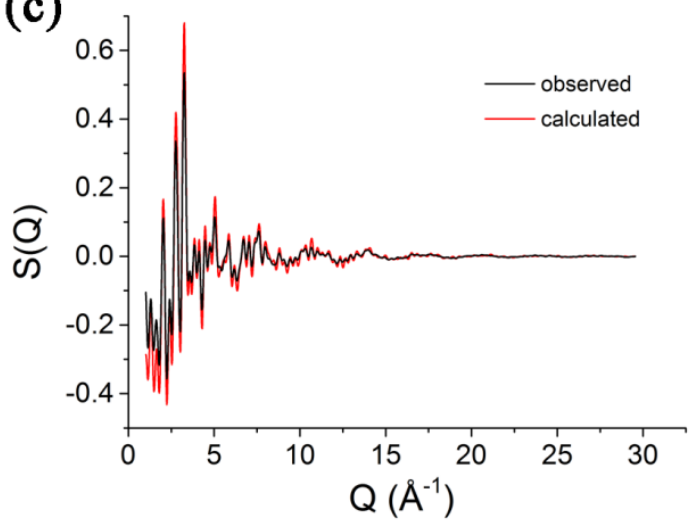

(b)

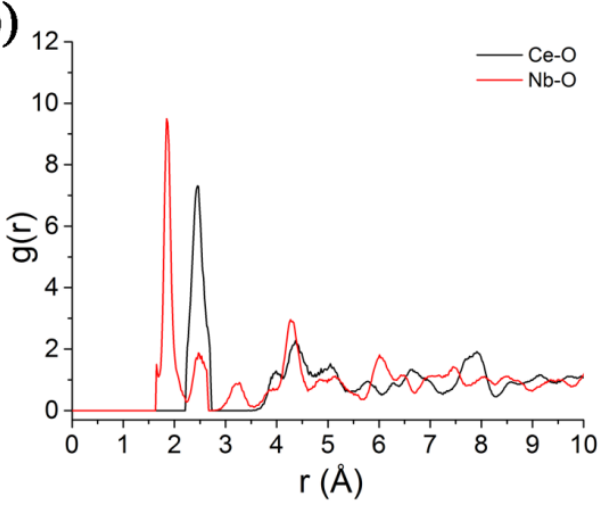

(d)

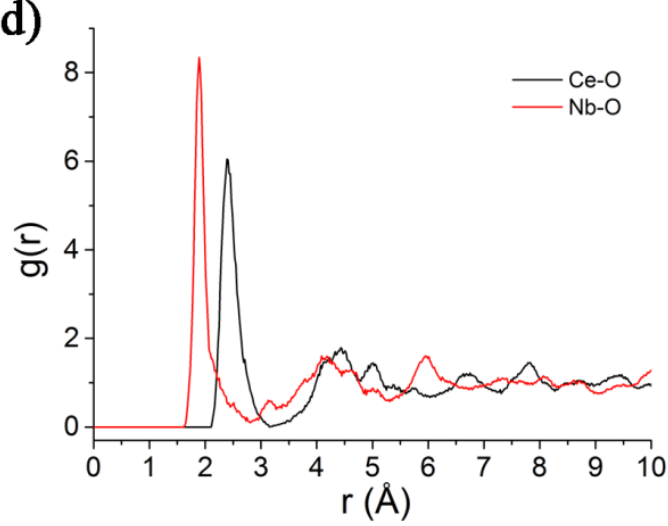

Figure 3: Pair distribution function analysis of $\mathrm{CeNbO}_{4+\delta}$ powders showing (a) total scattering structure factor $S(Q)$ and (b) partial pair correlation functions $g(r)$ of $\mathrm{CeNbO}_{4}$ and (c) $\mathrm{S}(\mathrm{Q})$ and $(\mathrm{d}) \mathrm{g}(\mathrm{r})$ of $\mathrm{CeNbO}_{4.25}$. For both cases $\mathrm{Ce}-\mathrm{O}$ and $\mathrm{Nb}-\mathrm{O}$ correlations were limited to $r \leq 10 \AA$. 
(a)

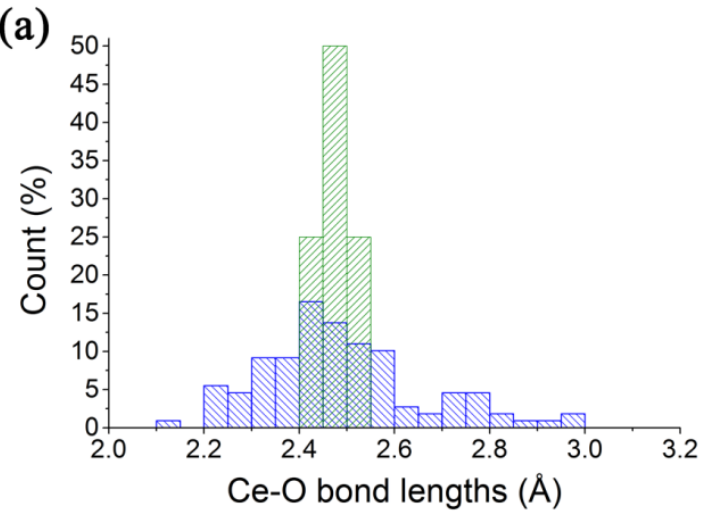

(c)

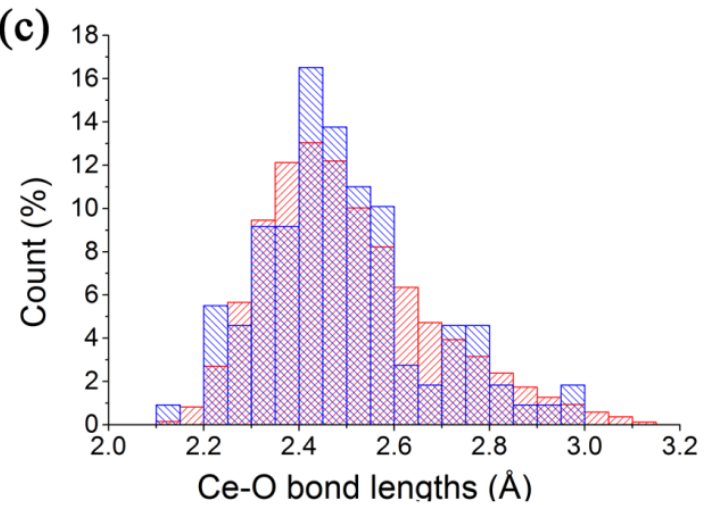

(e)

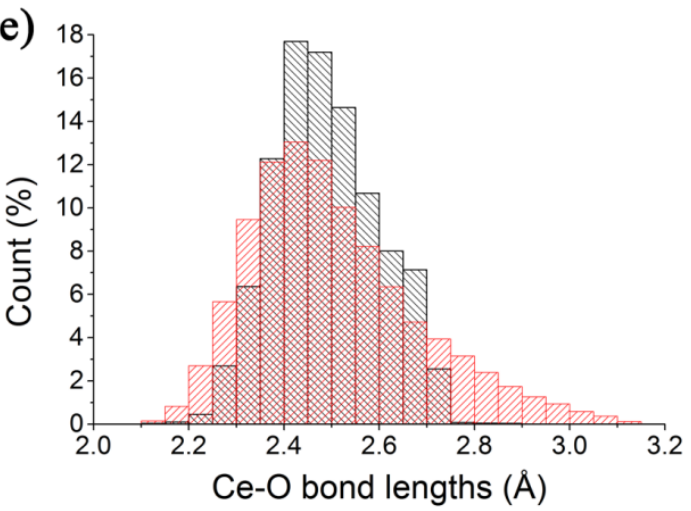

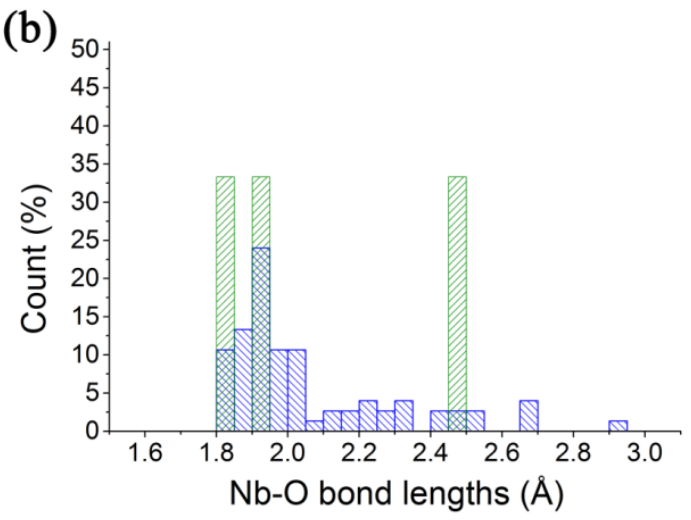

(d)

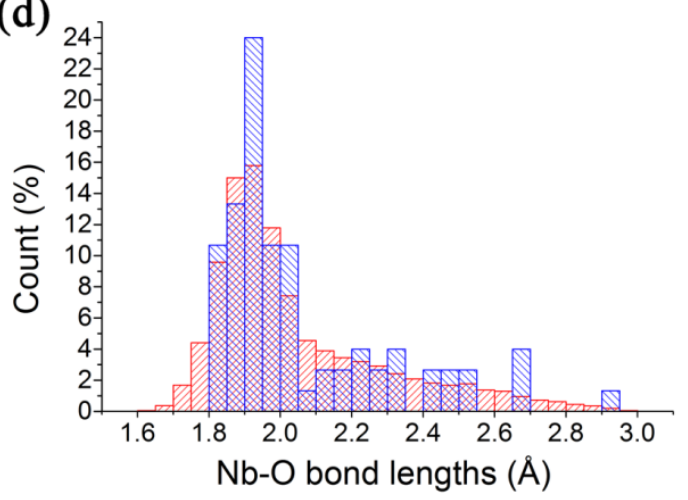

(f)

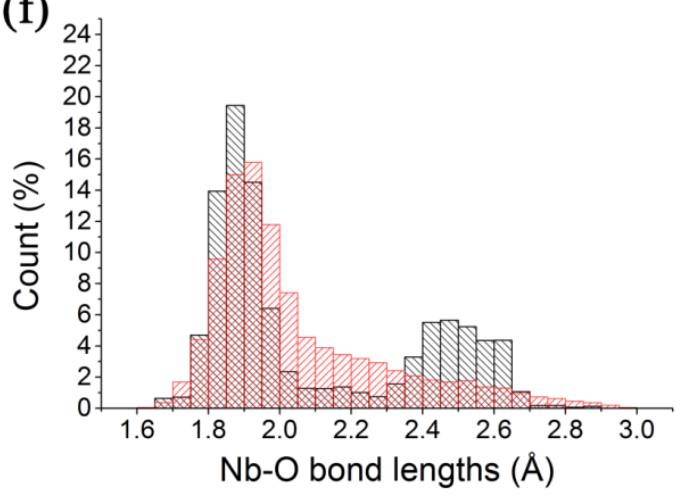

$\mathrm{CeNbO}_{4}$ - single crystal XRD

$\mathrm{CeNbO}_{4.25}$ - single crystal XRD

$\mathrm{CeNbO}_{4}-\mathrm{PDF}$

$\mathrm{CeNbO}_{4.25}$ - PDF

Figure 4: (a) $\mathrm{Ce}-\mathrm{O}$ and (b) $\mathrm{Nb}-\mathrm{O}$ bond length histogram of parent $\mathrm{CeNbO}_{4}{ }^{20}$ (green) and oxidized $\mathrm{CeNbO}_{4.25}$ (blue) derived from single crystal XRD showing a clear dispersion of the latter phase with the centroid located close to the parent phase. Total numbers of bond lengths for $\mathrm{Ce}-\mathrm{O}$ in $\mathrm{CeNbO}_{4}$ and $\mathrm{CeNbO}_{4.25}$ are 8 and 109; and $\mathrm{Nb}-\mathrm{O}$ are 6 and 75, respectively; (c) $\mathrm{Ce}-\mathrm{O}$ and (d) $\mathrm{Nb}-\mathrm{O}$ bond lengths histogram of $\mathrm{CeNbO}_{4.25}$ derived from single crystal XRD (blue) and PDF (red) with 27809 Ce-O bonds and 19105 Nb-O bonds for $\mathrm{CeNbO}_{4.25}$ - PDF; (e) $\mathrm{Ce}-\mathrm{O}$ and (f) $\mathrm{Nb}-\mathrm{O}$ bond lengths histogram of $\mathrm{CeNbO}_{4}$ (black) and $\mathrm{CeNbO}_{4.25}$ (red) derived from PDF with $32000 \mathrm{Ce}-\mathrm{O}$ bonds and $24053 \mathrm{Nb}-\mathrm{O}$ bonds for $\mathrm{CeNbO}_{4}$ - PDF. 
To confirm that the helical $\mathrm{Ce}^{3+} / \mathrm{Ce}^{4+}$ chains were favourable towards ion transport a molecular dynamics approach was employed. Initial lattice relaxation and optimization shows that better agreement between the experimental and calculated lattice parameters of $\mathrm{CeNbO}_{4.25}$ was achieved compared to the parent phase of $\mathrm{CeNbO}_{4}{ }^{20}$ with the same set of ionic potentials used. This suggests that the latter may not be a pure stoichiometric material, but rather, a slightly oxidized phase, whilst the former describes the positions of interstitial oxygens and the distinctive arrangement of $\mathrm{Ce}^{3+}$ and $\mathrm{Ce}^{4+}$ lattice sites. Figure 5 highlights the trajectory projections along the [010] and off-axis [001] directions showing the mean square displacement (MSD) of ions after 100 ps. The $\mathrm{CeNbO}_{4.25}$ sample clearly shows greater MSDs at $1073 \mathrm{~K}$ indicating that the oxygen ions were mobile along the $\mathrm{NbO}_{x}$ polyhedra slab. An enlarged trajectory plot calculated at the high temperature of $1573 \mathrm{~K}$ is shown in Figure $5 \mathrm{~g}$ clearly showing that the major migration pathway lies in the $x z$ plane, but with some connectivity observed along the $y$ crystallographic axis.
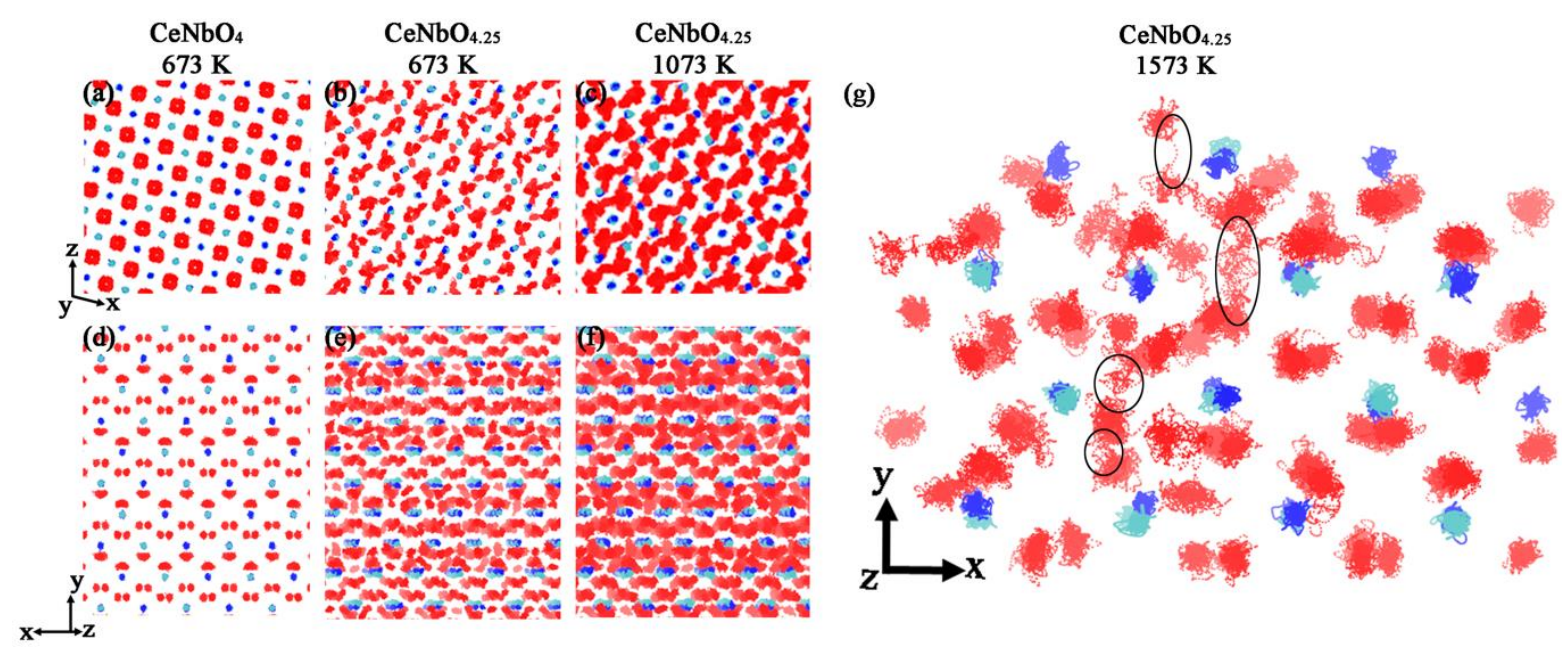

Figure 5: (a-c) [010] and (d-f) [001] rotated $18^{\circ}$ around $y$ trajectory projections after 100 ps MD simulation: (a,d) $\mathrm{CeNbO}_{4}$ at $673 \mathrm{~K}$, (b,e) $\mathrm{CeNbO}_{4.25}$ at $673 \mathrm{~K}$ and (c,f) $\mathrm{CeNbO}_{4.25}$ at $1073 \mathrm{~K}$ where light blue, dark blue and red represent $\mathrm{Ce}^{3+/ 4+}, \mathrm{Nb}^{5+}$ and $\mathrm{O}^{2-}$ ions respectively. Panel ( $\mathrm{g}$ ) is an enlarged trajectory at $1573 \mathrm{~K}$ clearly showing the connectivity along the $x z$ plane with the $y$ migration additionally observed and highlighted. 
Figures 6a and $\mathrm{b}$ compare the MSD of oxygen in $\mathrm{CeNbO}_{4.25}$ and $\mathrm{CeNbO}_{4}$ at different temperatures. The parent phase does not show diffusion within the timeframe used, whilst the oxidized phase readily allows diffusion. In an $N$ ion system, the diffusivity is given by:

$$
\frac{1}{N} \sum_{i=1}^{N}\left[r_{i}(t)-r_{i}(0)\right]^{2}=6 D t+B
$$

where $r_{i}(t)-r_{i}(0)$ is the displacement of an ion from its initial position after a time $t, D$ is the ionic diffusion coefficient, $t$ is time and $B$ is the displacement associated with the thermal vibrations around the equilibrium position.
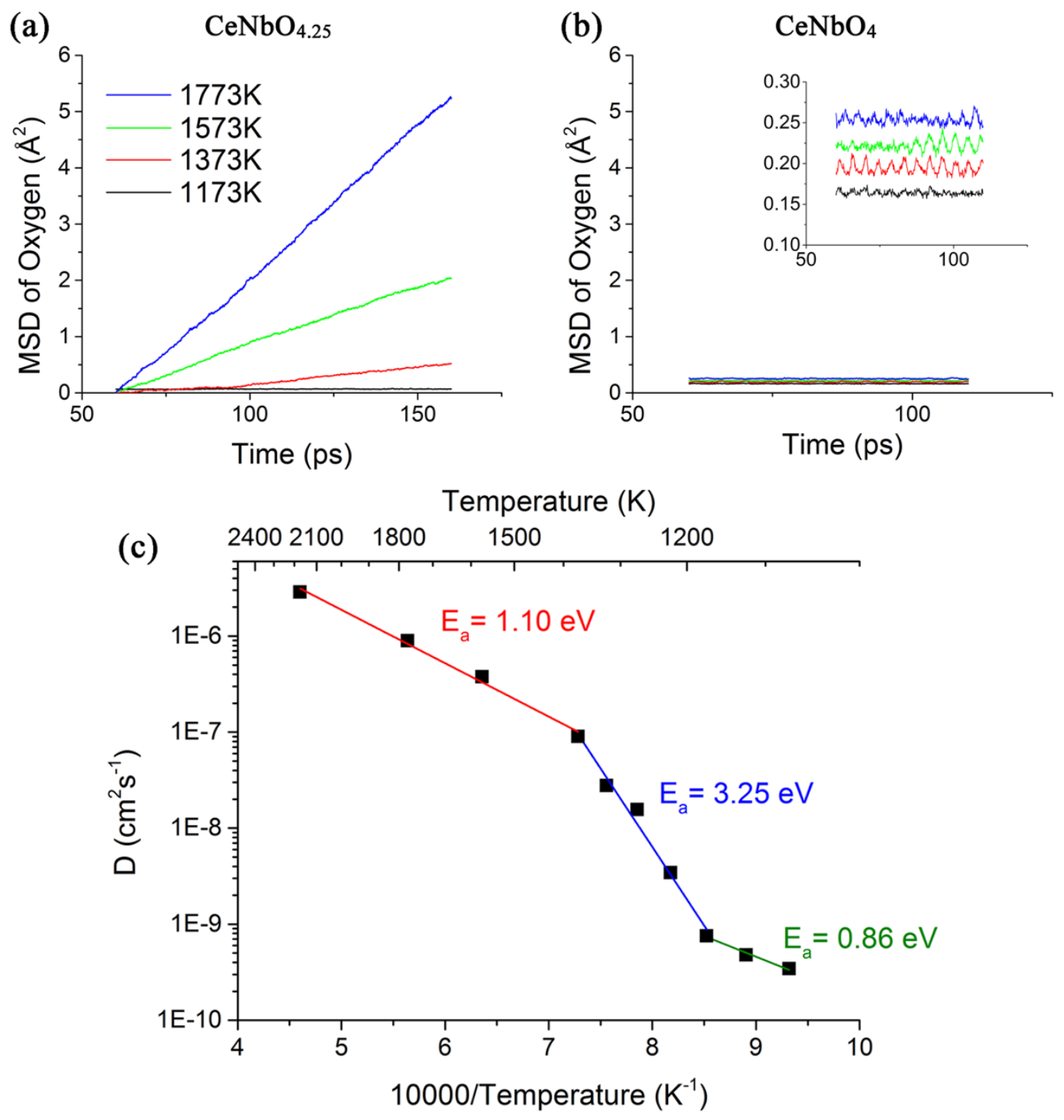

Figure 6: Mean square displacement of oxygen of (a) $\mathrm{CeNbO}_{4.25}$ and (b) $\mathrm{CeNbO}_{4}$ at different temperatures showing that the parent phase does not diffuse within the 


\section{timeframe used whilst the oxidized phase readily diffuses. (c) Oxygen diffusivity showing regions with different calculated activation energies.}

The calculated diffusion coefficients at $1073 \mathrm{~K}, 1573 \mathrm{~K}$ and $2173 \mathrm{~K}$ were $3 \times 10^{-10}, 4 \times 10^{-7}$ and $3 \times 10^{-6} \mathrm{~cm}^{2} \mathrm{~s}^{-1}$ respectively, with corresponding activation energies $\left(E_{a}\right)$ of $1.10 \mathrm{eV}(1373 \leq \mathrm{T} \leq$ $2173 \mathrm{~K})$ and $0.86 \mathrm{eV}(1073 \leq \mathrm{T} \leq 1173 \mathrm{~K})$ (Figure $6 \mathrm{c})$. At $1173 \mathrm{~K}$ the experimental value for $\mathrm{D}^{14}$ is about 2 orders of magnitude lower than our computed value, with $\mathrm{E}_{\mathrm{a}} 0.45 \mathrm{eV}$ lower. However, a higher value for $\mathrm{E}_{\mathrm{a}}$ of $0.99 \mathrm{eV}$ was reported by Packer and Skinner, ${ }^{15}$ which is comparable to this work. The difference in the activation energies may be associated with the transformation of the structure from commensurate oxygen excess $\mathrm{CeNbO}_{4.25}$ at low temperatures to the high temperature oxygen stoichiometric $\mathrm{CeNbO}_{4}$ phase. It is well known that the lowest temperature accessed by the MD calculations is at the point at where the phase transition occurs, and that the stoichiometric material is a poor oxygen ion conductor. This may also account for the difference in absolute oxygen diffusion coefficients. Another plausible explanation is that in the simulation, the MSD was averaged over all oxygen ions which may underestimate the mobile ion in $\mathrm{CeNbO}_{4.25}$. 


\section{CONCLUSIONS}

Commensurately modulated $\mathrm{CeNbO}_{4.25}$ with nominal oxygen excess has been shown to adopt a helical arrangement of $\mathrm{Ce}^{3+} \mathrm{O}_{\mathrm{x}}$ and $\mathrm{Ce}^{4+} \mathrm{O}_{\mathrm{x}}(8 \leq \mathrm{x} \leq 10)$ using a combination of single crystal and powder diffraction techniques, which leads to the anisotropic diffusion of oxygen along the $x z$ slab. The interstitial oxygen extends the coordination of $\mathrm{Nb}$ from a nominal 6 , within a $3 \AA$ bond shell distance, to 7 and 8 that serves to increase the connectivity between the " $\mathrm{NbO}_{6}$ " layers. The local environment of the $\mathrm{Nb}-\mathrm{O}$ and $\mathrm{Ce}-\mathrm{O}$ pairs deduced from pair distribution function analysis confirms this average distorted coordination environment. This serves as an alternative pathway for the conducting oxygen to cross from one slab to another along the $y$ direction whenever the oxide ion encounters a barrier, as seen in our molecular dynamics simulation at $1573 \mathrm{~K}$, whilst the parent $\mathrm{CeNbO}_{4}$ phase remains non-conducting even at higher temperature. These observations on this complex crystal structure offer new insights in the development of high performance materials for electrochemical devices, opening a wide compositional space that is no longer restricted to three-dimensional isotropic materials. 


\section{AUTHOR INFORMATION}

\section{Corresponding Author}

tbaikie@ntu.edu.sg; s.skinner@imperial.ac.uk

\section{Notes}

The authors declare no competing financial interest. 


\section{ACKNOWLEDGMENTS}

The authors gratefully acknowledge the support of the EPSRC for the award of a doctoral training account studentship for RDB. Additionally we acknowledge the support of King Abdullah University of Science and Technology who partially funded this work (SSP and JW). We further thank STFC for the award of neutron powder diffraction beam time at the Rutherford Appleton Laboratory (ISIS) under award RB1120177. We also acknowledge the Institut Laue-Langevin (ILL) for the award of Easy access on the D2B beamline and Diamond Light Source Ltd, UK, for access to the I11 powder diffraction beamline.

Supporting Information: The following data are included as supporting information: Table of refined single crystal X-ray diffraction data (S1), Table of refined atomic positions (S2), Table of selected bond lengths (S3), Table of Buckingham Potentials (S4), Table of experimental and calculated lattice parameters (S5), Micrograph of the single crystal (Fig S1), Model crystal structures (Figs S2 and S3), Refined diffraction data (Fig S4), refined total scattering data (Fig S5), pair distributions correlations from MD (Fig S6 \& S7) and associated references. 


\section{REFERENCES}

(1) Orera, A.; Slater, P. R. Chem. Mater. 2010, 22, 675.

(2) Kuang, X.; Green, M. A.; Niu, H.; Zajdel, P.; Dickinson, C.; Claridge, J. B.; Jantsky, L.; Rosseinsky, M. J. Nat Mater 2008, 7, 498.

(3) Wei, F.; Gasparyan, H.; Keenan, P. J.; Gutmann, M.; Fang, Y.; Baikie, T.; Claridge, J. B.; Slater, P. R.; Kloc, C. L.; White, T. J. J. Mater. Chem. A 2015, 3, 3091.

(4) Packer, R. J.; Barlow, J.; Cott, A.; Skinner, S. J. Solid State Ionics 2008, 179, 1094.

(5) Skinner, S. J.; Palmer, H. M.; Raj, E. S.; Kilner, J. A. J. Solid State Chem. 2004, $177,2685$.

(6) Negas, T.; Roth, R. S.; McDaniel, C. L.; Parker, H. S.; Olson, C. D. Mater. Res. Bull. 1977, 12, 1161.

(7) Roth, R. S.; Negas, T.; Parker, H. S.; Minor, D. B.; Jones, C. Mater. Res. Bull. 1977, 12, 1173 .

(8) Carter, S.; Selcuk, A.; Chater, R. J.; Kajda, J.; Kilner, J. A.; Steele, B. C. H. Solid State Ionics 1992, 53-56, 597.

(9) Minh, N. Q. J. Am. Ceram. Soc. 1993, 76, 563.

(10) Chroneos, A.; Yildiz, B.; Tarancon, A.; Parfitt, D.; Kilner, J. A. Energy Environ. Sci. 2011, 4, 2774.

(11) Kushima, A.; Parfitt, D.; Chroneos, A.; Yildiz, B.; Kilner, J. A.; Grimes, R. W. Phys. Chem. Chem. Phys. 2011, 13, 2242.

(12) Tsipis, E. V.; Munnings, C. N.; Kharton, V. V.; Skinner, S. J.; Frade, J. R. Solid State Ionics 2006, 177, 1015.

(13) Packer, R. J.; Skinner, S. J.; Yaremchenko, A. A.; Tsipis, E. V.; Kharton, V. V.; Patrakeev, M. V.; Bakhteeva, Y. A. J. Mater. Chem. 2006, 16, 3503.

(14) Packer, R. J.; Tsipis, E. V.; Munnings, C. N.; Kharton, V. V.; Skinner, S. J.; Frade, J. R. Solid State Ionics 2006, 177, 2059.

(15) Packer, R. J.; Skinner, S. J. Adv. Mater. 2010, 22, 1613.

(16) Thompson, J. G.; Withers, R. L.; Brink, F. J. J. Solid State Chem. 1999, 143, 122.

(17) Skinner, S. J.; Kang, Y. Solid State Sci. 2003, 5, 1475.

(18) Santoro, A.; Marezio, M.; Roth, R. S.; Minor, D. J. Solid State Chem. 1980, 35, 167.

I37.

(19) Skinner, S. J.; Brooks, I. J. E.; Munnings, C. N. Acta Crystallogr. C 2004, 60,

(20) Bayliss, R. D.; Pramana, S. S.; An, T.; Wei, F.; Kloc, C. L.; White, A. J. P.; Skinner, S. J.; White, T. J.; Baikie, T. J. Solid State Chem. 2013, 204, 291.

(21) Cava, J. R.; Roth, R. S. AIP Conf. Proc. 1979, 53, 361.

(22) Cava, R. J.; Roth, R. S.; Negas, T.; Parker, H. S.; Minor, D. B. J. Solid State Chem. 1981, 40, 318.

(23) Skinner, S. J.; Packer, R. J.; Bayliss, R. D.; Illy, B.; Prestipino, C.; Ryan, M. P. Solid State Ionics 2011, 192, 659.

(24) Petříček, V.; Dušek, M.; Palatinus, L. Z. Kristallogr 2014, 229, 345.

(25) Palatinus, L.; Chapuis, G. J. Appl. Cryst. 2007, 40, 786.

(26) Bruker; Bruker AXS Inc.: Madison, Wisconsin, USA, 2008.

(27) Larson, A. C.; Dreele, R. B. V. General Structure Analysis System (GSAS), Los Alamos National Laboratory, 2004.

(28) Soper, A. K.; Barney, E. R. J. Appl. Crystallogr. 2011, 44, 714.

(29) McGreevy, R. L.; Pusztai, L. Mol. Simul. 1988, 1, 359. 
(30) McGreevy, R. L. Nucl. Inst. Meth. Phys. A 1995, 354, 1.

(31) Matthew, G. T.; David, A. K.; Martin, T. D.; Andrew, L. G.; Qun, H. J. Phys.: Condens. Matter 2007, 19, 335218.

(32) Born, M.; Mayer, J. E. Z. Phys. 1932, 75, 1.

(33) Ewald, P. Ann. Phys. 1921, 64, 253.

(34) Buckingham, R. A. Proc. R. Soc. London, A 1938, 168, 264.

(35) Smith, W.; Forester, T. R. J. Mol. Graphics 1996, 14, 136.

(36) Todorov, I. T.; Smith, W.; Trachenko, K.; Dove, M. T. J. Mater. Chem. 2006, $16,1911$.

(37) Swope, W. C.; Andersen, H. C.; Berens, P. H.; Wilson, K. R. J. Chem. Phys. 1982, 76, 637.

(38) Gale, J. D. J. Chem. Soc., Faraday Trans. 1997, 93, 629.

(39) Humphrey, W.; Dalke, A.; Schulten, K. J. Mol. Graphics 1996, 14, 33.

(40) Brese, N. E.; O'Keeffe, M. Acta Crystallogr. B 1991, 47, 192.

(41) Brown, I. D.; Altermatt, D. Acta Crystallogr. B 1985, 41, 244.

(42) Hahn, T.; Looijenga-Vos, A. In International Table for Crystallography; Hahn, T., Ed.; Springer: Heidelberg, 2006; Vol. A.

(43) Swanson, H. E.; Tatge, E. Standard X-ray diffraction powder patterns, National Bureau of Standards, Washington, D.C., USA, 1953.

(44) Pramana, S. S.; Klooster, W. T.; White, T. J. Acta Crystallogr. B 2007, 63, 597.

\section{TABLE OF CONTENTS (TOC) GRAPHIC}
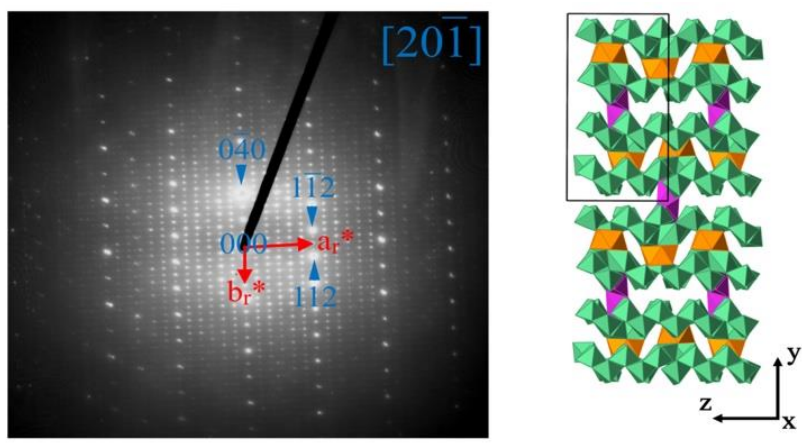